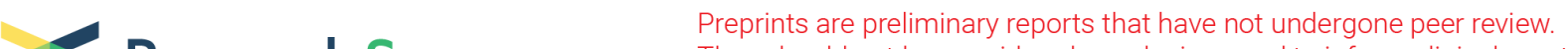

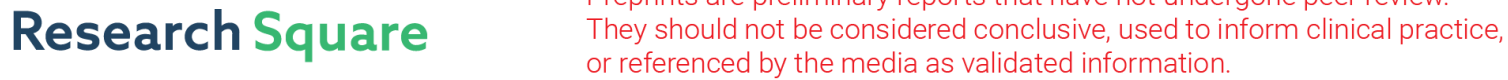 \\ Epidemic Trend of Dental Fluorosis in Mainland China, 1995-2020: A Systematic Review and Meta- Analysis
}

\section{Yuzhen Zhan}

Chongqing Medical University Stomatology College: Stomatological Hospital of Chongqing Medical University

\section{Lianjie Xiong}

Chongqing Medical University Stomatology College: Stomatological Hospital of Chongqing Medical University

\section{Zhoujie Gong}

Chongqing Medical University Stomatology College: Stomatological Hospital of Chongqing Medical University

\section{Ting Xu}

Chongqing Medical University Stomatology College: Stomatological Hospital of Chongqing Medical University

\section{Xiaonan Zhang ( $\nabla$ zhangxiaonan@hospital.cqmu.edu.cn )}

Chongqing Medical University Stomatology College: Stomatological Hospital of Chongqing Medical University https://orcid.org/0000-0003-0155-776X

\section{Research Article}

Keywords: Epidemiology, Dental fluorosis, China, Oral health survey, Systematic review, Meta-analysis

Posted Date: May 7th, 2021

DOI: https://doi.org/10.21203/rs.3.rs-423131/v1

License: (c) (i) This work is licensed under a Creative Commons Attribution 4.0 International License. Read Full License 


\section{Abstract}

The aim of this system review and meta-analysis was to explore the epidemiological characteristics of dental fluorosis in mainland China from 1995 to 2020. A comprehensive literature search was conducted through PubMed, Embase, CBM, CNKI, Chinese Wan Fang database, and VIP database. Subgroup analyses were done to explore epidemic tread of dental fluorosis (gender, location, survey year and geographical distribution) with the help of relative software. Forty-one publications were included in this study. The overall prevalence was $23.6 \%$, and the prevalence of dental fluorosis increased from $18.8 \%$ during $1995-1999$ to $34.3 \%$ during $2010-2014$, while it decreased to $20.5 \%$ during $2015-2019$. There was no significant difference in prevalence between boys $(15.7 \%)$ and girls $(15.2 \%)(\mathrm{RR}=1.05,95 \% \mathrm{Cl}$ : 1.02-1.07); and the prevalence of dental fluorosis in rural areas (14.5\%) was slightly higher than those in urban areas $(12.7 \%)(\mathrm{RR}=0.93,95 \% \mathrm{Cl}$ : $0.76-1.13)$. The prevalence before $(63.3 \%)$ and after $(34.7 \%)$ water improvement showed a great benefit of fluoride reduction policy. Result of this meta-analysis provides evidence enable governments taking effective measures to control dental fluorosis.

\section{Introduction}

Dental fluorosis (DF) is a specific esthetic disturbance, which is caused by excessive intake of fluoride during the development of teeth(Zhang et al. 2013). Clinically, the manifestations of DF could be divided into three degrees: in mild situations, the enamel surface shows white and opaque; in moderate conditions, spots may be brownish (AL et al. 2009); and in severe cases, enamel is fragile which can result in fracture and loss of tissue (FDC et al. 2017). Due to unsightly appearance and discoloration of teeth, people with dental fluorosis are associated with some psychological problems such as interpersonal sensitivity, compulsion, depression and hostility ( $N$ et al. 2017, Zou et al. 2016), which has a negative impact on the individuals' OHRQoL (oral health-related quality of life) (SS et al. 2018). Besides, dental fluorosis affects the reconstruction of the alveolar bone, the movement of teeth and the adhesion between teeth and brackets (Dong \&Zhang 2016, Yu et al. 2016), which increases the difficulty in orthodontic treatment. The treatment of dental fluorosis includes micro-abrasion, external bleaching, full crown and ceramic veneers. In moderate and severe cases, ceramic veneers as the best choice cost about $50,000 \mathrm{RMB}$ accounting for half of the annual per capita disposable income of Chinese population (AM et al 2018). Hence, dental fluorosis has become a crucial public health issue because of its influences on the aesthetic and economic of individuals.

In China, the second, third and fourth oral epidemiological surveys showed that the national prevalence of dental fluorosis was $6.89 \%$ in 1995, 11.7\% in 2005 and 14.4\% in 2015 respectively, showing an increasing trend. Besides, the prevalence of dental fluorosis in China is variable across the country. The prevalence of dental fluorosis is relatively low in some places, while the prevalence of dental fluorosis is particularly high in some areas where the fluoride content in drinking water is too high. For instance, the prevalence of DF was 9.58\%(Fan et al. 2008) in Yunnan, while 35.17\%(Zhu et al. 2006) in Qingdao. 
It has been reported that the content of fluorine resources in China accounts for $60 \%$ of the total reserves in the world (Luo 2011), which probably affects the prevalence of DF. Moreover, China is a vast and multiethnic country with diverse geographical environment. Thus, the causes of dental fluorosis in China are more complicated than other countries. For instance, people of some western minority areas in China have the habit of drinking brick tea that contains high content fluorine. Long-term intaking high fluorine content brick tea causes the endemic fluorosis, which manifested as dental fluorosis (La et al. 2016). Additionally, exhaust gas from coal burning may pollute local air, water and crops (Chen et al. 2004, Dong et al. 2011). Therefore, it is necessary to figure out the epidemiological characteristics of dental fluorosis in China, which would be benefit to allocate the medical resources effectively in China.

Currently, there are no systematic reviews on dental fluorosis in adolescents in mainland China. Therefore, adolescents aged 8-12 years were selected for this study. We analysed the epidemiological characteristics of dental fluorosis from all epidemiological survey articles in this meta-analysis, to explore the prevalence trend with time, gender, location, and geography. This may provide useful information for the prevention of DF and implementation of relevant oral health policies for those populations.

\section{Methodology}

\section{Data sources and searches}

Six online databases were comprehensively searched by the first and second author for the studies published before December, 2020 (PubMed, Embase, CBM, CNKI, Chinese Wan Fang Database, and VIP database). The keywords used for the search included "dental", "tooth or teeth", "enamel", "fluoride or fluorosis", "China or Chinese", "prevalence or incidence" and "epidemiology". Moreover, potentially relevant articles that may be missed were identified through manual searching of reference lists. The systematic review and meta-analysis were reported according to the Preferred Reporting Items for Systematic Reviews and Meta-Analyses (PRISMA) guidance(Moher et al. 2009).

\section{Literature selection}

Inclusion criteria: the articles selected in this study were according to the following inclusion criteria; (1) surveys done in mainland China (except for Taiwan, Hong Kong, and Macao); (2) were written in English or Chinese; (3) participants focused on children aged between 8 and 12 ; (4) reported prevalence and risk factors as primary articles; (5) provided full text.

Exclusion criteria: the articles excluded were: (1) letters to editors, conference abstract and presentation, case reports, master and doctoral theses; (2) researches based on the special populations (for example, based on patients); (3) articles were not fully accessed; (4) studies didn't report geographic locations.

\section{Data extraction and quality assessment}

Study selection and data extraction were carried out by the first and second author independently. In the first stage, eligible citations were selected by screening the headlines and abstracts; in the second stage, 
the full-text analysis was performed. Cohen's Kappa was used to check the consistency between examiners. Disagreements were resolved by consensus and by the third author when necessary. For each study, the items of data extraction included the name of the first author, year of publication, geographical location and territorial levels, survey date, sampling method, diagnostic criteria, the sample size (and proportion of DF patients), the prevalence of DF and DFI (dental fluorosis index). If surveying date was not stated in the article, we assumed it as 2 years before publication.

We used the Strengthening the Reporting of Observational Studies in Epidemiology (STROBE) to assess the quality of included studies (Supplementary Checklist 1). This tool consists of 22 items (32 sub-items) that are considered necessary for excellent reporting of observational studies. Quality assessments of all included articles were assessed by answering 'yes or no' to each item in the STROBE checklist. The assessment was judged by two independent authors, and any discrepancy of their assessment results was resolved by consensus or by the third author.

\section{Statistical analysis}

Pooled estimates of prevalence and $95 \%$ confidence intervals $(\mathrm{Cl})$ were calculated using STATA software version 15.1. The heterogeneity was checked using Q-test and $\mathrm{I}^{2}$ test. The level of heterogeneity was low $\left(I^{2}<50 \%\right.$ or $\left.\mathrm{P}>0.1\right)$, a fixed-effects model was adopted. Otherwise, the random-effects model would be selected. We used funnel plots and Begg's test to observe potential publication bias across the studies (Fig.7), the result was considered to be significant when $P \leq 0.05$. In addition, subgroup analyses were performed based on gender, survey year, province and region of studies. Cochrane Review Manager (RevMan) version 5.3 was used to estimate the Relative Risk (RR) and 95\% Cl between different subgroups. In the sensitivity analysis, we eliminated one article individually and the summary results were not affected, indicating that the results were robust enough. Furthermore, studies were sorted into five periods to observe the temporal trends of DF: 1995-1999, 2000-2004, 2005-2009, 2010-2014, $\geq 2015$. Finally, we created a prevalence map to reflect spatial distribution in mainland China using ArcGIS10.2 software.

\section{Results}

A total of 8663 records were initially retrieved by keywords search on Embase ( $n=1814)$, PubMed $(n=2341)$, CNKI $(n=1241)$, the Wan Fang $(n=1787)$, the VIP $(n=1398)$ and CBM $(n=79)$. The second, third, fourth National Oral Health Survey of our country were included as well. After the exclusion of duplicate articles (2136) and irrelevant references (6527) from the pooled database by screening the title and abstracts, a full text review was conducted for 827 studies. In total, 786 articles did not meet the eligibility criteria, in which 86 did not state age clearly or age is not within 8-12, 3 were conducted in quota sampling, 30 studies did not have available data, 638 studies reported irrelevant information, 11 studies did not state survey site, 18 publications repeated survey time and sites. In the end, 41 studies are included in our systematic review and meta-analysis (Fig.1). They comprise 3 national-level, 12 provincial-level, 19 city-level, and 7 county-level studies, which involve 22 provinces, four municipalities 
and four autonomous regions of the mainland China (Chen et al. 2014, Chen et al. 2004, Chen et al. 2007, Deng et al. 2011, Dong et al. 2011, Fan et al. 2008, Fan et al. 2012, Hu 2008, Huang et al. 2008, Jiang et al. 2010, Li et al. 2004, Li et al. 2012, Li et al. 2018, Liang et al. 2008, Ma \&Wei 1998, Ma et al. 2008, Shao et al. 2004, Shen et al. 2007, Shen et al. 2013, Song et al. 2004, Wu et al. 2010, Wu 2010, Xu et al. 2004, Yan et al. 2012, Yao \&Wang 2016, Yun et al. 2005, Zhai et al. 2017, Zhang et al. 2017, Zhang et al. 2014, Zhang 2003, Zhao et al. 2017, Zhou et al. 2011, Zhu et al. 2009, Zhu et al. 2006)

The weighted Kappa statistic for examiner consistency during the title and abstract screening was 0.74 , and 0.79 in the full-text analysis (Supplementary Table S1). The description of the 41 included studies was summarized in Table 1. Among these, prevalence rates of DF varied from $3.1 \%$ to $86.5 \%$. The highest prevalence rate (86.5\%) was reported in Yunnan and lowest prevalence rate (3.1\%) was reported in Guangxi. Dean method defined by the World Health Organization (WHO) was used as the diagnostic criteria of dental fluorosis in 40 studies and all 41 surveys recruited dentists and trained examiners as investigators. As for quality assessment, the number of positive answers ('yes') for the 32 listed items on the Strobe checklist for each study was at least 24 (Supplementary Table S2), which indicating that the quality of the 41 eligible studies was satisfactory.

\section{Prevalence of DF in mainland China}

Prevalence of DF over time. The overall estimates of DF prevalence in mainland China was $23.6 \%$ (95\% Cl: $19.3-28.0 \%$, Table 2) using a random-effects model, which involved 433322 participants. A total of 41 studies reported the prevalence of DF during 1995-2020. The prevalence of DF in survey year groups of 1995-1999, 2000-2004, 2005-2009, 2010-2014 and 2015- were 18.8\% (95\% Cl: 4.7\%-42.4\%), 29.9\% (95\% Cl: $25-34.8 \%), 16.2 \%$ (95\% Cl: 9.1\%-23.3\%), 34.3\% (95\% Cl: 25.1-43.5\%), 20.5\% (95\% Cl: 8.9-32.1\%) respectively. Fig. 2 illustrated an overall slightly ascending trend over time across mainland China, while an apparent reduction in prevalence of DF was observed in 2005-2009 and an apparent increasing in prevalence of DF was observed in 2010-2014.

Prevalence of DF by gender. A total of 17 articles reported prevalence of DF estimates by gender, yielding an overall prevalence of DF in boys and girls were $15.7 \%$ (95\% Cl: $11.9 \%-19.5 \%)$ and $15.2 \%(95 \% \mathrm{Cl}$ : $11.6 \%-18.8 \%$ ) respectively. The final pooled meta-analysis indicated no statistically significant difference in the prevalence of DF between the sexes (RR=1.05, 95\% Cl: 1.02-1.07, Fig.3).

Prevalence of DF by area. A total of 13 articles reported DF prevalence in children aged 8-12 from both urban and rural. The pooled prevalence of DF in rural and urban China was 14.5\% (95\% Cl: 10.6-18.3\%) and $12.7 \%$ (95\% Cl: $10.2-15.2 \%)$ respectively. Furthermore, higher prevalence estimates of DF were found in rural, while lower prevalence in urban (RR=0.93, 95\% Cl: 0.76-1.13, Fig. 4).

Prevalence of DF by water improvement. A total of 7 articles reported DF prevalence in children aged 8-12 years from both water improvement before (WIB) and water improvement after (WIA). The pooled prevalence of DF from WIB and WIA was 63.3\% (95\% Cl: 57-69.6\%) and 34.7\% (95\% Cl: 31.3-38.1\%) 
respectively. Furthermore, the prevalence of DF for WIB was significantly higher than that for WIA ( $\mathrm{RR}=1.76,95 \% \mathrm{Cl}$ : 1.61-1.93, Fig. 5).

\section{Geographical distribution of DF prevalence in mainland China}

Figure 6 demonstrates a color-coded map of the distribution of the dental fluorosis in mainland China (data available from most provinces, except Tibet). Five different color distribution areas were created on the map based on the prevalence of DF. And significant variation was observed for geographical region across provinces. The highest prevalence zone showed on the map in the darkest red, including Tianjin (39.9\%), Chongqing (41.8\%) and Jiangsu (42.5\%). And the prevalence of DF in Guangdong (36.5\%) and Inner Mongolia (33.0\%) was substantial high, as well as in Qinghai, Hebei, Shandong. Other provinces show low prevalence relatively.

\section{Discussion}

Dental fluorosis acting as the earliest indicator of excess fluoride exposure in population was an important public oral health issue around the world (Idon \&Enabulele 2018). The meta-analysis is the first of these kinds to estimate the epidemiological characteristics of dental fluorosis in mainland China.

In this meta-analysis, the prevalence of dental fluorosis has increased considerably from 2009 to 2018, while a reduction of prevalence during 2005-2009 was shown in our study. The possible reason is more likely due to the implementation of the fluorine reduction and water improvement policy in 1997 (Lu et al. 2011). As the result of meta-analysis shows, the prevalence of dental fluorosis has decreased significantly since 2005. It suggests that the prevalence of dental fluorosis decreased obviously after eight years' water improvement, which is consistent with other studies (Chen et al. 2004, Sun et al. 2017). Meanwhile, the overall results of the 7 studies included demonstrated significant reduction in DF prevalence after water improvement. Hence, the fluoride reduction policy is the most effective measure to reduce the prevalence of dental fluorosis. Moreover, thanks to researchers report on dental fluorosis, the government has paid more and more attention to oral hygiene and adopts corresponding anti-fluorination measures actively.

Nevertheless, it was worth noting that there is an ascent trend of prevalence during 2010-2014. Two possible reasons can explain this trend: the failure of water improvement project and the increase in rate of reports on the prevalence of DF. Firstly, during the implementation of the water improvement project, the water modification equipment was damaged gradually, which caused the leakage of high-fluorine water (Yin et al. 2017). In a study conducted in Zhoukou (a city in Henan province) (Hao 2010), 57.25\% of the water improvement project were damaged or scrapped, which finally resulted in a water fluoride exceeding rate of $34.84 \%$. Similar situations have been reported in other studies (Cao et al. 2009, Qiao et al. 2014, Zhao et al. 2013). Secondly, in recent decades, more and more researchers investigated the prevalence of dental fluorosis in various regions of China. Relevant reports increased dramatically. Besides, the examiner's diagnosis of dental fluorosis might be more precise than before, which can diagnose more patients with DF. Thus, our research results show an ascent trend eventually. The 
continued increase in fluorosis rates in mainland China indicates that additional measures need to be implemented to reduce the prevalence.

Of our studies conducted, people in rural areas are more likely to suffer from dental fluorosis than those in urban. The possible reason is that the waste gas from coal burning enters into drinking water or crops (Yao \&Wang 2016), and another is that rural residents consumed water from the well or groundwater with high fluorine content (Wang et al. 2013).

The geographical map shows significant regional differences. As the map shows, the prevalence rates of DF in Tianjin, Chongqing and Jiangsu are relatively high. In China, endemic fluorosis mainly includes drinking water-type endemic fluorosis, coal-burning endemic fluorosis and tea-type endemic fluorosis. Drinking water-type endemic fluorosis keeps main position (Chen \&Yu 2013). Research also indicates that dental fluorosis in Tianjin is a drinking water-type of endemic fluorosis with a serious historical conditions (Sun et al. 2001). In spite of good results got from water improvement, the prevalence of dental fluorosis remains high because the water improvement project has not been working properly in recent years (Cui et al. 2017). The disease situation in some areas is rather complicated like Chongqing. Dental fluorosis in Chongqing is both drinking water-type and coal-burning type. The combined effect of two different causes exacerbates the prevalence of dental fluorosis. In the light of the above reasons, it suggests that the government should control the disease according to local conditions. For drinking-water type endemic fluorosis, the policy of water improvement should be implemented actively. In drinking teatype fluorosis areas, government should strengthen anti-fluoride education to eliminate the harm of drinking brick tea. For coal-burning fluorosis areas, the stove should be changed to reduce coal combustion.

Several limitations should be considered into our findings. Firstly, the literature reporting on dental fluorosis in China was insufficient, even though we finally captured 41 articles in our meta-analysis. Secondly, some areas like Tibet lack relevant articles for prevalence of DF. Hence, we are not able to assess the prevalence of DF among the general populations of China. Moreover, a high level of heterogeneity was observed between studies. That might be attributed to eating habits, family economic status and genetic susceptibility, but we did not have enough data to explain. Finally, the diagnosis of dental fluorosis was mainly dependent on the investigators' proficiency. In another word, bias from investigators still exist. For example, mild dental fluorosis and enamel hypoplasia are difficult to differentiate. We hope that there will be more epidemiological studies on DF in China.

\section{Conclusion}

Although it is not possible to determine the prevalence of dental fluorosis in mainland China through these studies; nonetheless, the epidemiological characteristics of dental fluorosis in mainland China increased first and then decreased cannot be ignored. And findings show that the prevalence of dental fluorosis has been greatly reduced after the water improvement policy. Result of this meta-analysis provides evidence enable governments taking effective measures to control dental fluorosis. The 
government should continue to strengthen the fluoride reduction and water reform policy, and strengthen the maintenance of water improvement engineering equipment.

\section{Declarations}

Authors' contributions Xiaonan Zhang and Yuzhen Zhan contributed to the study conception and design. Study material preparation, data collection and analysis were performed by Yuzhen Zhan, Lianjie Xiong, Zhoujie Gong, Ting Xu, Xiaonan Zhang. All authors have written, read and approved this manuscript for submission.

Funding This work was supported by Chongqing Medical Reserve Talent Studio for Young People, Grant/Award Number: ZQNYXGDRCGZS2019004.

Data availability All data generated or analyzed during this study are included in this published article (and its supplementary information files).

\section{Compliance with ethical standards}

Conflicts of interest The authors declare that they have no conflict of interest.

Ethical approval Not applicable.

Content to participate Not applicable.

Content to publish Not applicable.

\section{References}

1. AL B, DM L, PK D (2009): The impact of fluoride on ameloblasts and the mechanisms of enamel fluorosis. Journal of dental research $88,877-93$

2. AM EM (2018): Aesthetic Rehabilitation of a Severe Dental Fluorosis Case with Ceramic Veneers: A Step-by-Step Guide. Case reports in dentistry 2018, 4063165

3. Cao Y, Xie Y, Dong J, Li A, Mu W (2009): Prevalence of fluorosis associated with drinking water in Dali county,Shaanxi,2008. Disease surveillance 24, 722-723

4. Chen G, Yu B (2013): Advances in research on molecular biological mechanism of endemic skeletal fluorosis. Chinese Journal of Endemiology 32, 470-472

5. Chen P, He D, Wei S, Pu G, La C, Jiang H, Li S, Lu Q, Zhao Y (2014): A cross-sectional investigation of drinking brick-tea fluorosis of children aged 8-12 in Qinghai Province. Chinese Journal of Endemiology 33, 53-55

6. Chen S, Xu H, Xu L, Li B, Zou Y, Wang J, Yu J (2004): Analysis of Prevalence of Dental Fluorosis and Fluoride Content in the Drinking-water After Water Improvement to Reduce Fluoride in Drinking-water High Fluoride Areas in Chaoyang Guangdong Province. Chinese Primary Health Care 18, 41-44 
7. Chen W, Huang S, Zhang J (2007): Dental Fluorosis in a 12 Years old Guangdong Cohort: A Sampled Investigation in 2005. Journal of Dental Prevention and Treatment 15, 266-267

8. Cui Y, Liu Z, Li W, Wang Y, Hou C (2017): Investigation on the drinking-water-born endemic fluorosis in Tianjin in 2016. China Preventive Medicine 18, 814-818

9. Deng Z, Chen S, Tian H, Zhao Y (2011): Survey on fluoride in drinking-water and dental fluorosis of children in Hancheng ancient district of Xi'an city. Chinese Journal of Child Health Care 19, 582-584

10. Dong J, Zhou X, Wu T, Shan J, Li Y (2011): An analysis of the incidence of dental Fluorosis of children aged 8 to 12 years in endemic fluorosis villages that changed water in Lianyungang city. Chinese Journal of Control of Endemic Diseases 26, 278-280

11. Dong J, Zhang Z (2016): Effects of bonding after microabrasion with normal acid etching time on losing ratio of orthodontic brackets to dental fluorosis. Chinese Journal of Endemiology 35, 772-775

12. Fan Q, Wang B, Wang W, Li Q, Zhang C, Li Y, Xu W, Gu X (2008): Sampling survey report on fluorosis in 12-year-old population in Yunnan Province in 2005. Journal of Dental Prevention and Treatment $16,514-515$

13. Fan Q, Yao S, Ji J (2012): Survey of the Incidence of Dental Fluorosis in High Fluoride Areas of Zhaotong, Yunnan Province after Stove Changing and Decreasing of Fluorine. Journal of Oral Science Research 28, 961-963

14. FDC A-D, F M-C, AC C-V, J F-H (2017): Prevalence of dental fluorosis in Mexico 2005-2015: a literature review. Salud publica de Mexico 59, 306-313

15. Hao J (2010): Analysis of epidemiological survey results of endemic fluorosis in Zhoukou City. Henan Journal of Preventive Medicine 21, 433-434

16. Hu Y (2008): Epidemiological survey of fluorosis in 12-year-old population in Qinghai province. Chinese Journal of School Health 29, 844-845

17. Huang H, Deng H, Li Z, Liu G, Lin Y, Huang G, Li X, He K (2008): Sampling survey of fluorosis in 12year-old children in Guangxi Zhuang Autonomous Region in 2005. Journal of Guangxi Medical University 25, 320-321

18. Idon PI, Enabulele JE (2018): Prevalence, severity, and request for treatment of dental fluorosis among adults in an endemic region of Northern Nigeria. Eur J Dent 12, 184-190

19. Jiang L, Lin J, Wang J, Zhang H, Zhou Z, Hu Y, Wang L, Liu Q, Liu Y (2010): Sampled investigation for epidemiology of dental fluorosis among 12-year-old children in Chongqing. Chongqing Medicine 39, 1237-1238

20. La C, Wei S, Chen P, Yang P, Jiang H, Li S, Zhao Y, Ma J, Zhang Q (2016): Analysis of a survey results on drinking brick-tea type fluorosis in Hainan Prefecture of Qinghai Province in 2013. Chinese Journal of Endemiology 35, 132-134

21. Li B, Wang J, Yu J, Yang C, Chen S, Xu L (2004): Epidemiological Analysis of Dental Fluorosis Prevalence in the Children After Water Improvement Based Reduction of Fluoride in the Fluorosis Areas in Guangdong Province. Chinese Primary Health Care 18, 38-41 
22. Li Q, Liu J, Zhang C, Yi Y, Tang Q, Yan K, Duan M (2012): Investigation on the prevalence of dental fluorosis in children aged 8-12 years in Kaiyang County, Guizhou Province in 2009. Journal of Modern Medicine and Health 28, 3198-3199

23. Li Q, Zhao Z, Chen P, Yang P, Zhan P (2018): An investigation and analysis of the prevalence of drinking-tea type fluorosis in Hainan Prefecture, Qinghai Province in 2016. Chinese Journal of Endemiology 37, 319-322

24. Liang X, Jin L, Li H, Wang Y, Song Y, Wu Y, Wang G (2008): Investigation on Prevalence of Dental Fluorosis among Children in Huhhot. China Preventive Medicine 9, 891-893

25. Lu J, Lu W, Fan G, Chen H (2011): Monitoring and analysis of water and fluoride reduction engineering in drinking-water type fluorisis area in Quanzhou City in 2010. Strait Journal of Preventive Medicine 17, 53-54

26. Luo X (2011): Research on the current development status of fluorine resources in China. China New Technologies and New Products, 38

27. Ma B, Wei X (1998): Investigation of dental fluorosis before and after fluoride reduction in Ningcheng County. Chinese Journal of Epidemiology, 63

28. Ma L, Tian M, Yu X (2008): Sampling survey report on dental fluorosis in 12 and 15 years old in Guiyang city. Journal of Dental Prevention and Treatment 16, 323-324

29. Moher D, Liberati A, Tetzlaff J, Altman DG, Group P (2009): Preferred reporting items for systematic reviews and meta-analyses: the PRISMA statement. PLoS Med 6, e1000097

30. N M-F, M N-R, A N-R, R G-G, ME I-C, L S-P, S L-V, R B-M (2017): Impact of Dental Fluorosis, Socioeconomic Status and Self-Perception in Adolescents Exposed to a High Level of Fluoride in Water. International journal of environmental research and public health 14

31. Qiao X, Shi H, Xing Y (2014): Shangqiu drinking water type fluorosis monitoring village children aged 8 to 12 dental fluorosis prevalence survey analysis. Henan Journal of Preventive Medicine, 286-288

32. Shao Y, Zhang X, Yang D (2004): Survey of incidence of dental fluorosis in fluorosis endemic areas in Changnan County. China Tropical Medicine 4, 628-629,631

33. Shen J, Gu H, Wang Y, Zhou H (2007): Prevalence of Dental Caries and Dental Fluorosis Among Students Aged 12 Years in Jiangsu Province. Chinese Journal of School Health 28, 721-722,724

34. Shen W, Ma L, Tian K, Tian M (2013): Investigation report of dental fluorosis in students aged $12 \otimes 15$ in Xifeng of Guiyang city. Practical Journal of Clinical Medicine 10, 156-158

35. Song J, Nie B, Jiang P (2004): Survey of dental fluorosis in children aged 12 and 15 in parts of Xinjiang. Endemic Diseases Bulletin『china) 19, 61-62

36. SS M, ML G, KS C, FC S, FF D (2018): Dental Fluorosis Treatment Can Improve the Individuals' OHRQoL? Results from a Randomized Clinical Trial. Brazilian dental journal 29, 109-116

37. Sun D, Shen Y, Zhao X, Wei H, Zhang X, Sun Y, Yu G (2001): Analysis on the disease trend and present state of endemic fluorosis in China mainland. Chinese Journal of Endemiology 20, 429-433 
38. Sun Y, Zhang M, Zhang H, Wang P, Zhang W (2017): Relationship between water improvement time in drinking-water type fluorosis area and prevalence of dental fluorosis in children. Jiangsu Journal of Preventive Medicine 28, 685-686

39. Wang E, Zheng Z, Liu W, Wang J (2013): Analysis of monitoring results of children dental fluorosis in drinking-water-borne endemic fluorosis areas in Liaoning province in 2011. Chinese Journal of Endemiology 32, 183-185

40. Wu X, Li X, Gan B, Chen Y, Zheng X, Yang D (2010): Analysis on Epidemic Situation of Dental Fluorosis in Shimen County. Practical Preventive Medicine 17, 2419-2420

41. Wu Y (2010): Sampling survey of fluorosis in 12-year-old and 15-year-old population in Kuitun District in 2009. Endemic Diseases Bulletin囚china) 25, 35,37

42. Xu L, Xu H, Chen S, Li B, Yu J, Wang J (2004): Epidemiological survey and analysis of fluoride content in drinking water and dental fluorosis prevalence water-type high fluoride areas in Shantou city,Guangdong province. Chinese Rural Health Service Administration 24, 38-40

43. Yan J, Zhong Z, Wang Y, Luo X, Yan W, Mou L (2012): An investigation of dental fluorosis of children aged 8 - 12 in Wushan and Fengjie counties of Chongqing. Chinese Journal of Endemiology 31, 423425

44. Yao Q, Wang W (2016): Investigation on the prevalence of dental fluorosis in coal-burning endemic fluorosis areas in Luoyang City from 2011 to 2014. Journal of Medical Pest Control 32, 301-303

45. Yin Y, Gao J, Zhang B, Wang Y, Yun Z, Chen P (2017): Surveillance on drinking water-borne endemic fluorosis in Shandong province from 2009 to 2014. Journal of Environment and Health 34, 345-349

46. Yu Y, Zou D, Liu X, Yu X, Yang X, Ying J (2016): The effects of dental fluorosis on the tooth movement in adolescents after tooth extraction for orthodontic treatment. Journal of Practical Stomatology 32, 77-80

47. Yun Z, Chen P, Bian J, Qin Q, Wang Y, Gao H, Ma A, Zhao L, Hao J (2005): Comparative analysis on child dental fluorosis under different circumstances of water-improvement and defluoridation in Shandong Province. Chinese Journal of Endemiology 24, 659-661

48. Zhai L, Li L, Wang K, Gao J, Zhang B, Li H, Yun Z, Wang X, Chen P (2017): Epidemiological investigation on endemic fluorosis of drinking water type in Ningjin, Xiajin and Wucheng counties in the northwest area of Shandong Province. Chinese Journal of Endemiology 36, 731-735

49. Zhang B, Li D, Zhang N, He P, Hu X, Wang X (2014): A epidemiological study of children's dental fluorosis in coal-burning-borne endemic fluorosis areas of three counties in Guizhou Province. Chinese Journal of Endemiology 33, 167-169

50. Zhang B, An D, Li D, Yao D, Zhang N, Ye H, Gao J, Hu X, Wang X (2017): An epidemiological investigation of dental fluorosis of children aged 8-12 in coal-burning type endemic fluorosis areas in Guizhou Province. Chinese Journal of Endemiology 36, 269-273

51. Zhang J, Deng J, Hui Z, Pan K (2013): A Study of the Enamel Surface Morphology and Morphologic Changes after Cold Light Whitening through Scanning Electron Microscope. Progress in Modern Biomedicine 13, 2928-2935 
52. Zhang M (2003): Analysis of the effect of preventing dental fluorosis in Chengde City from 1982 to 2001. Chinese Journal of Endemiology 22, 255-255

53. Zhao L, Wang C, Gao Y, Sun D (2013): National annual monitoring report of drinking-water-borne endemic fluorosis in 2010 and 2011. Chinese Journal of Endemiology 32, 177-182

54. Zhao Z, Li Q, Yang P, Chen P (2017): Investigaion on the situation of drinking-tea type fluorosis in children aged 8-12 years in Yushu Prefecture, Qinghai Province in 2016. Chinese Journal of Endemiology 36, 931

55. Zhou Q, Liu J, Zhang C, Zhang S, Li Y (2011): Epidemiology survey of dental caries and fluorosis of children in Kunming city. West China Journal of Stomatology 29, 514-516

56. Zhu M, Han Y, Zhang H, Hou W, Yi X, Wu D (2009): A sampled survey of dental health of 12-year-old chiidren in Beijing area in 2005. Beijing Journal of Stomatology 17, 281-285

57. Zhu W, Lu X, Wang Y, Li P, Gao H, Ma A (2006): Investigation on Children's Dental Fluorosis in Endemic Fluorosis in Qingdao City. Preventive Medicine Tribune 12, 537-538

58. Zou D, Yu Y, Zhao W, Wang L, Liu G (2016): Study on influence of dental fluorosis on adolescents' mental health. Chinese Nursing Research 30, 1718-1720

\section{Tables}

Table 1. Description of the included 41 studies. NOHES: National Oral Health Epidemiology Survey; NA: not available; DFI: dental fluorosis index. 


\begin{tabular}{|c|c|c|c|c|c|c|c|c|c|}
\hline $\begin{array}{c}\text { First } \\
\text { author\& published } \\
\text { year }\end{array}$ & City & Territorial levels & Survey date & Sampling method & Diagnostic criteria & Sample size & cases & DFI & prevalence \\
\hline Chen et a1.2014 & Qinghai & Provincial & 2010 & Random sampling & Dean & 12355 & 3012 & 0.44 & 24.38 \\
\hline Hu et a1.2008 & Qinghai & Provincial & 2005 & Random sampling & Dean & 791 & 315 & 0.76 & 39.82 \\
\hline Yan et a1.2012 & Chongaing & County & 2010 & Random sampling & Dean & 38209 & 16466 & 0.599 & 43.09 \\
\hline Jiang et a1.2010 & Chongaing & City & $2005-2006$ & Random sampling & Dean & 749 & 49 & 0.13 & 6.54 \\
\hline Shen et a1.2013 & Guizhou & County & 2011 & Random sampling & Dean & 104 & 16 & 0.29 & 15.38 \\
\hline Ma et a1.2008 & Guizhou & City & 2003 & Random sampling & Dean & 1100 & 247 & 0.46 & 22.45 \\
\hline Zhang et a1.2014 & Guizhou & Provincial & 2010 & Random sampling & Dean & 1727 & 323 & NA & 18.7 \\
\hline Deng et a1.2011 & Shanxi & City & 2010 & Random sampling & Dean & 457 & 177 & 0.16 & 38.73 \\
\hline Wuet a1.2010 & Hunan & County & 2009 & Random sampling & Dean & 1754 & 106 & 0.111 & 6.04 \\
\hline Shen et a1.2007 & Jiangsu & Provincial & 2005 & Random sampling & Dean & 792 & 185 & 0.33 & 23.39 \\
\hline Jiet al.2012 & Yunnan & City & 2012 & Random sampling & Dean & 400 & 346 & 2.12 & 86.5 \\
\hline Yao et a1.2016 & Henan & City & $2011-2014$ & Random sampling & Dean & 4109 & 613 & 0.28 & 14.92 \\
\hline Wuet a1.2010 & Xinjiang & County & 2009 & Random sampling & Dean & 409 & 107 & 0.46 & 26.16 \\
\hline Huang et a1.2008 & Guangxi & Provincial & 2005 & Random sampling & Dean & 773 & 24 & 0.23 & 3.1 \\
\hline Chen et a1.2007 & Guangdong & Provincial & 2005 & Random sampling & Dean & 720 & 32 & 0.03 & 4.44 \\
\hline Zhu et a1.2009 & Beijing & City & 2005 & Random sampling & Dean & 792 & 27 & 0.1 & 3.41 \\
\hline Fan et a1.2008 & Yunnan & Provincial & 2005 & Random sampling & Dean & 720 & 69 & 0.09 & 9.58 \\
\hline Zho et a1.2006 & Shandong & City & 2004 & Random sampling & Dean & 5939 & 2086 & 0.69 & 35.17 \\
\hline Song et a1.2004 & Xinjiang & Provincial & 2002 & Random sampling & Dean & 1475 & 139 & $\mathrm{NA}$ & 9.42 \\
\hline NOHES.1995 & Mainland & Country & 1995 & Random sampling & Dean & 23452 & 1615 & 0.169 & 6.89 \\
\hline NOHES.2005 & Mainland & Country & 2005 & Random sampling & Dean & 23464 & 2743 & 0.25 & 11.7 \\
\hline NOHES.2015 & Mainland & Country & 2015 & Random sampling & Dean & 27821 & 4013 & 0.28 & 14.4 \\
\hline Zhou et a1.2011 & Yunnan & Ciry & 2009 & Random sampling & Dean & 1149 & 47 & 0.03 & 4.1 \\
\hline Chen et a1.2004 & Guangdong & Ciry & 2001 & Random sampling & Dean & 23553 & 8482 & NA & 36.01 \\
\hline Dong et a1.2011 & Jiangsu & Ciry & 2006 & NA & Dean & 20814 & 9211 & $\mathrm{NA}$ & 44.25 \\
\hline Li et a1.2004 & Guangdong & Provincial & $2001-2003$ & NA & Dean & 43802 & 18215 & NA & 41.58 \\
\hline Zhang et a1.2003 & Hebei & Ciry & 2001 & NA & Dean & 189 & 32 & 0.27 & 16.93 \\
\hline Yun et a1.2005 & Guangdong & Provincial & 2001 & Random sampling & Dean & 5103 & 1669 & 0.73 & 32.71 \\
\hline Ma et a1.1998 & Neimenggu & Coungty & 1996 & NA & Dean & 952 & 294 & NA & 30.88 \\
\hline $\mathrm{Xu}$ et a1.2004 & Guangdong & Ciry & 2002 & NA & Dean & 24349 & 8639 & NA & 35.48 \\
\hline Li et a1.2012 & Guizhou & County & 2009 & NA & Dean & 27588 & 2824 & 0.13 & 10.24 \\
\hline Liang et a1.2008 & Neimengru & Ciry & 2005 & NA & Dean & 8092 & 2779 & NA & 34.34 \\
\hline Shao et a1.2004 & Zhejiang & County & 2003 & Random samoling & $\begin{array}{l}\text { Three-degree } \\
\text { nine-tvoe method }\end{array}$ & 2680 & 1010 & $\mathrm{NA}$ & 37.69 \\
\hline Zhang et a1.2017 & Guizhou & Provincial & 2014 & Random sampling & Dean & 17962 & 5803 & NA & 32.31 \\
\hline Li et a1.2018 & Hainan & City & 2016 & Random sampling & Dean & 3975 & 559 & 0.24 & 14.06 \\
\hline Zhao et a1.2017 & Qinghai & City & 2016 & Random sampling & Dean & 4790 & 1562 & 0.55 & 32.61 \\
\hline $\mathrm{Xu}$ et a1.2018 & Shandong & City & 2016 & Random sampling & Dean & 32420 & 1964 & 0.14 & 6.06 \\
\hline Wang et a1.2019 & Jilin & Provincial & 2018 & Random sampling & Dean & 960 & 293 & NA & 30.5 \\
\hline Lan et a1.2020 & Guangdong & City & 2018 & Random sampling & Dean & 1232 & 57 & 0.1 & 4.63 \\
\hline Dong et a1.2018 & Shanxi & City & 2017 & Random sampling & Dean & 929 & 211 & 0.4 & 22.71 \\
\hline Liv et a1.2018 & Tianiin & Citv & 2017 & Random samoling & Dean & 64671 & 25395 & 0.73 & 39.27 \\
\hline
\end{tabular}

Table 2. Pooled prevalence of dental fluorosis in mainland China during 1995-2020. WIB: water improvement before; WIA: water improvement after; Cl: confidence interval. 


\begin{tabular}{|c|c|c|c|c|c|}
\hline & Number of study & Sample size & Cases & Pooled prevalence(\%) & Prevalence(\%) $95 \% \mathrm{CI}$ \\
\hline Overall prevalence & 41 & 433322 & 121756 & 23.6 & $19.3-28.0$ \\
\hline \multicolumn{6}{|l|}{ Time period } \\
\hline $1995-1999$ & 2 & 24404 & 1909 & 18.8 & $4.7-42.4$ \\
\hline $2000-2004$ & 9 & 108190 & 40519 & 29.9 & $25.0-34.8$ \\
\hline $2005-2009$ & 14 & 88607 & 18518 & 16.2 & $9.1-23.3$ \\
\hline $2010-2014$ & 8 & 75323 & 26756 & 34.3 & $25.1-43.5$ \\
\hline $2015-$ & 8 & 136798 & 34054 & 20.5 & $8.9-32.1$ \\
\hline \multicolumn{6}{|l|}{ Location } \\
\hline Urban & 13 & 46576 & 4200 & 12.7 & $10.2-15.2$ \\
\hline Rural & 13 & 37473 & 5548 & 14.5 & $10.6-18.3$ \\
\hline \multicolumn{6}{|l|}{ Gender } \\
\hline Male & 17 & 72302 & 10246 & 15.7 & $11.9-19.5$ \\
\hline Female & 17 & 68857 & 9432 & 15.2 & $11.6-18.8$ \\
\hline \multicolumn{6}{|l|}{ Change water } \\
\hline before & 7 & 142908 & 91851 & 63.3 & $57.0-69.6$ \\
\hline after & 7 & 118762 & 46542 & 34.7 & $31.3-38.1$ \\
\hline
\end{tabular}

\section{Supplemental Files}

Supplemental Files are not available with this version

\section{Figures}




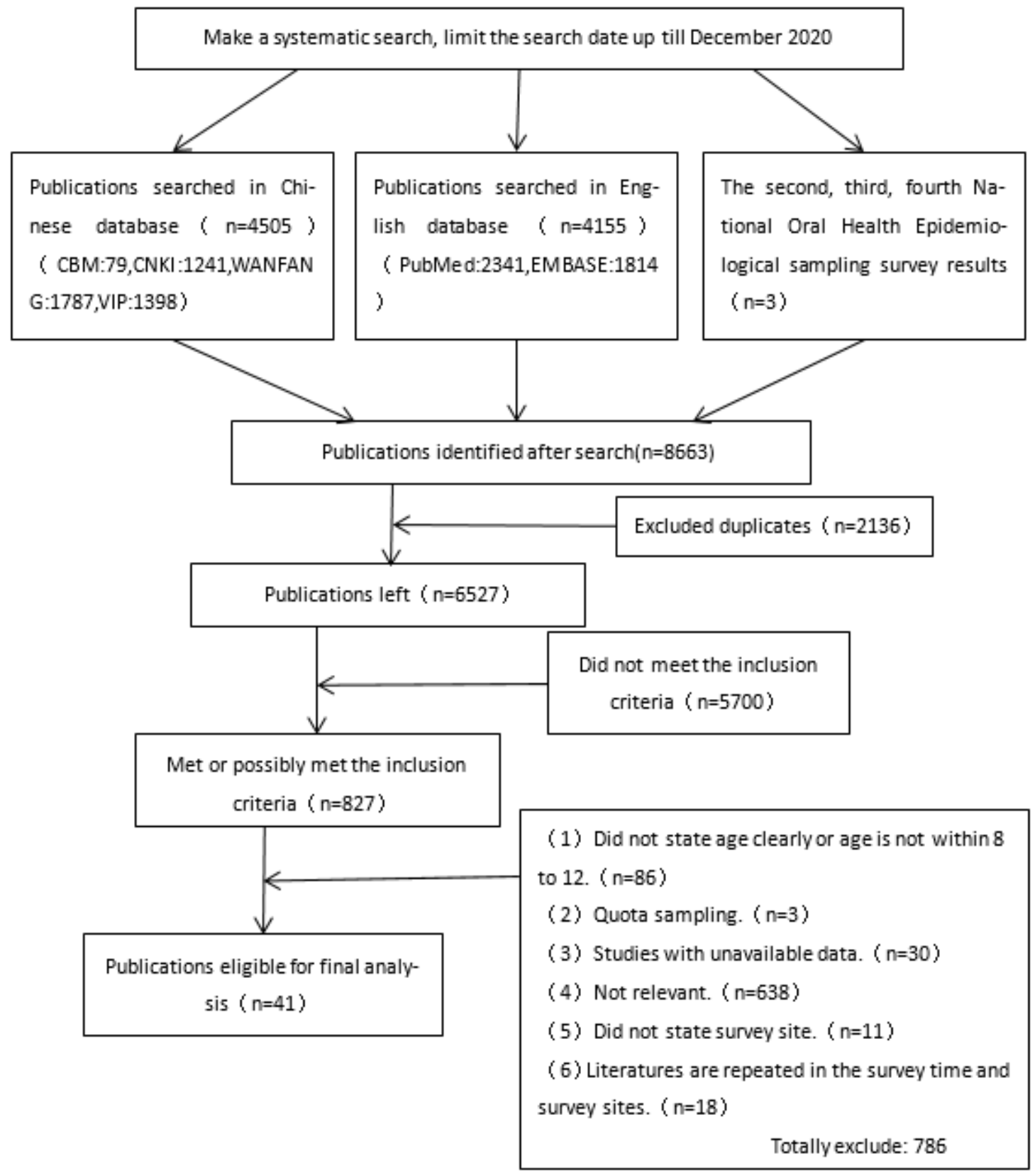

\section{Figure 1}

Flow chart of search strategy and literature selection.

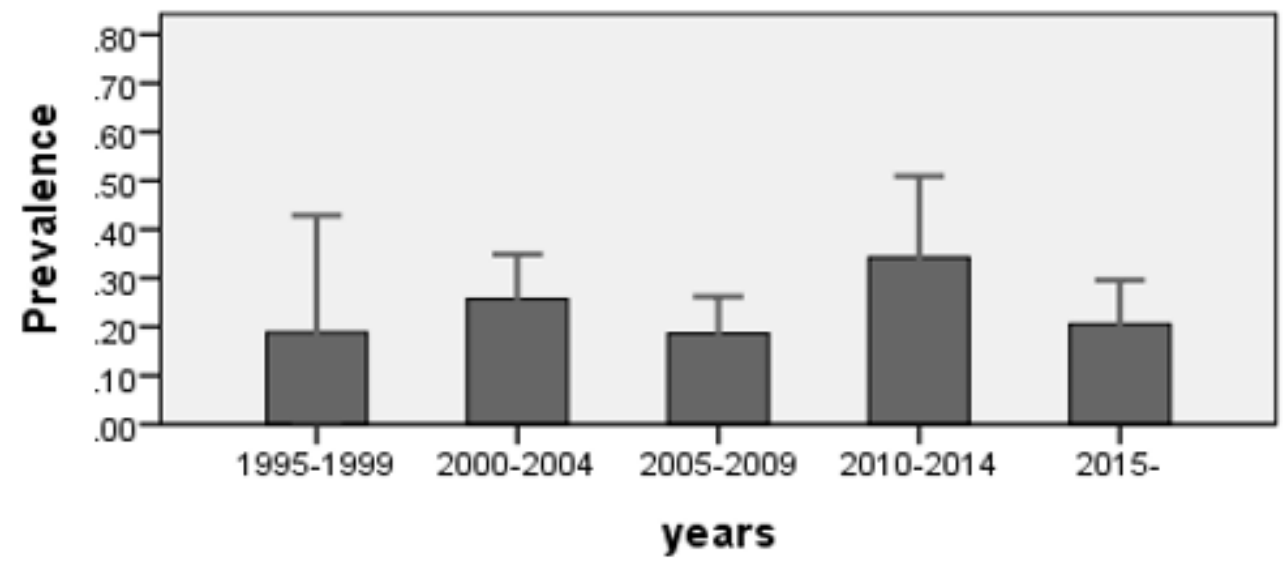

Page 15/18 
Figure 2

Temporal trends of the prevalence of dental fluorosis in mainland China during 1995-2020.

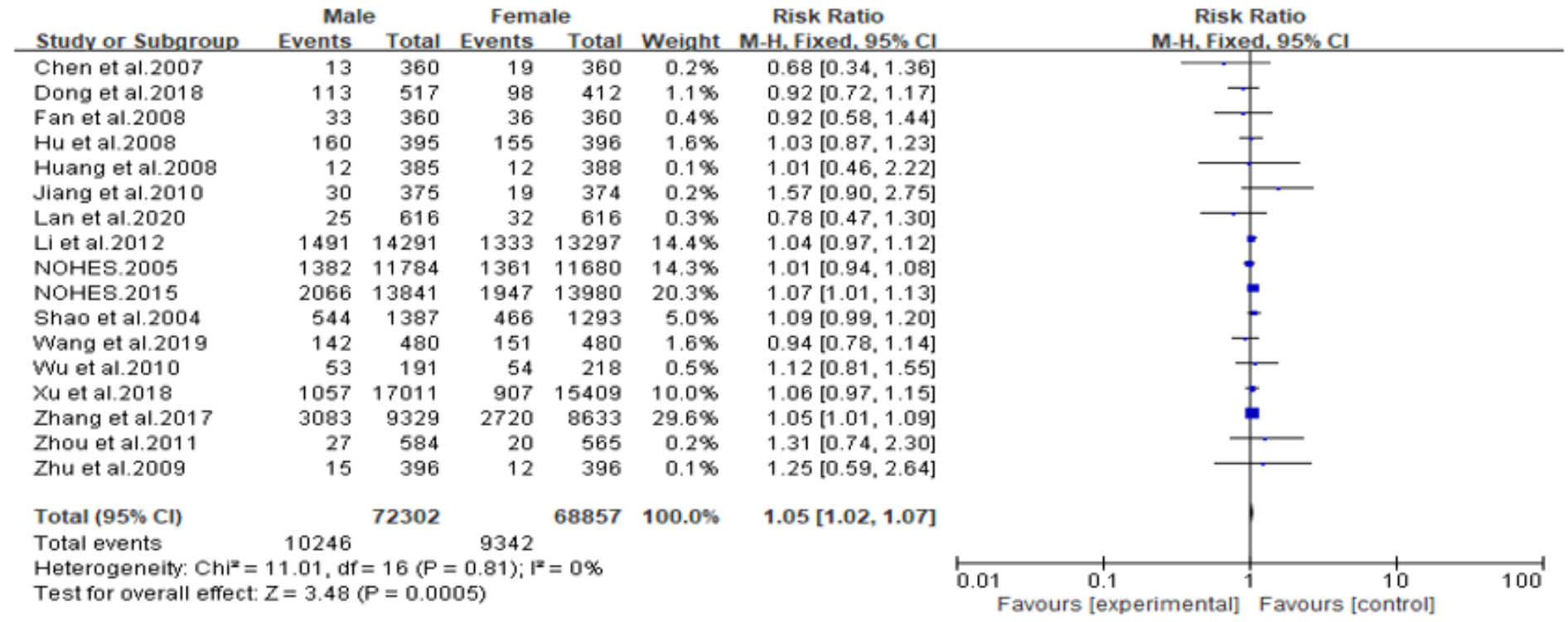

\section{Figure 3}

Forest plot of dental fluorosis among different gender in mainland China during 1995-2020.

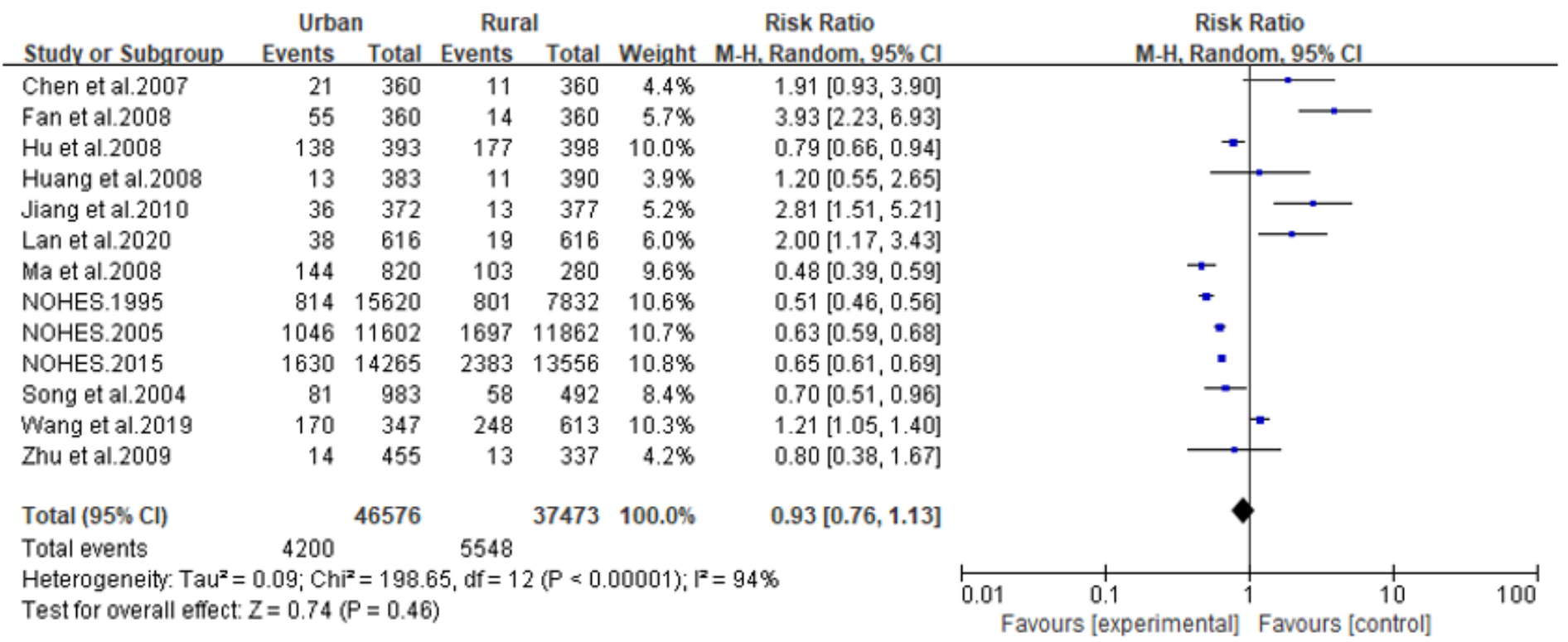

\section{Figure 4}

Forest plot of dental fluorosis among rural and urban areas of mainland China during 1995-2020. 


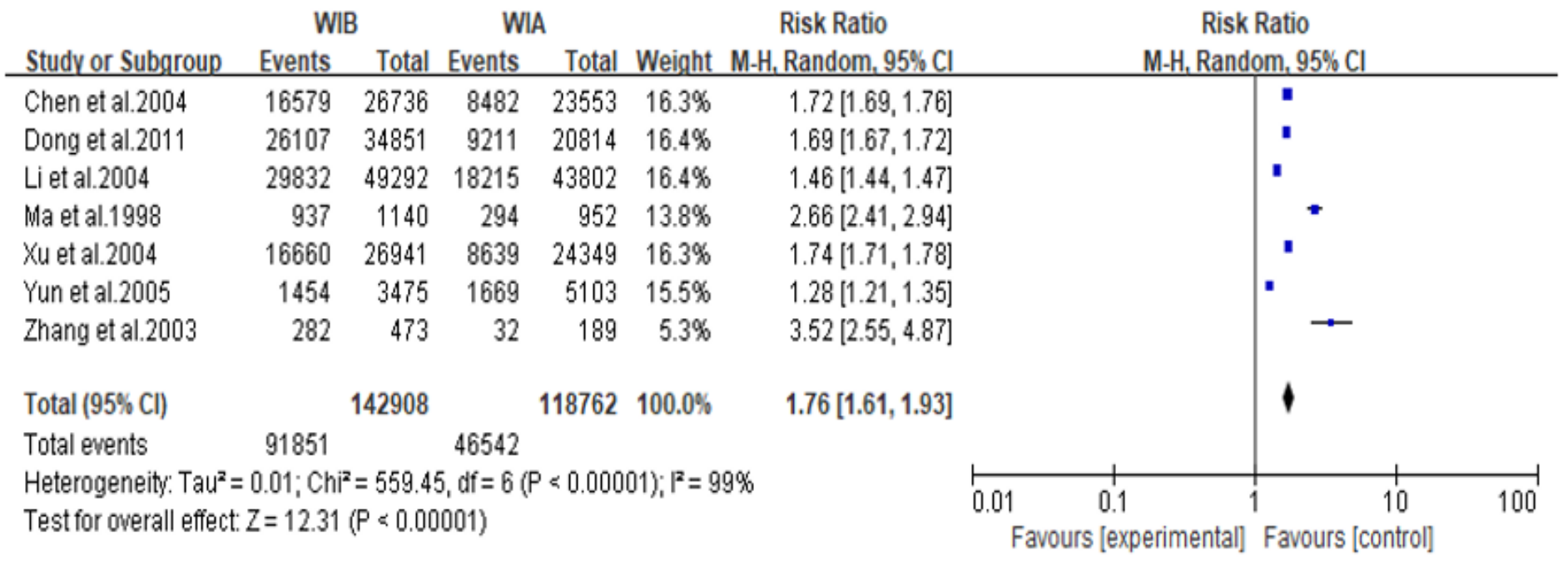

\section{Figure 5}

Forest plot of dental fluorosis among WIB (water improvement before) and WIA (water improvement after) of mainland China during 1995-2020.

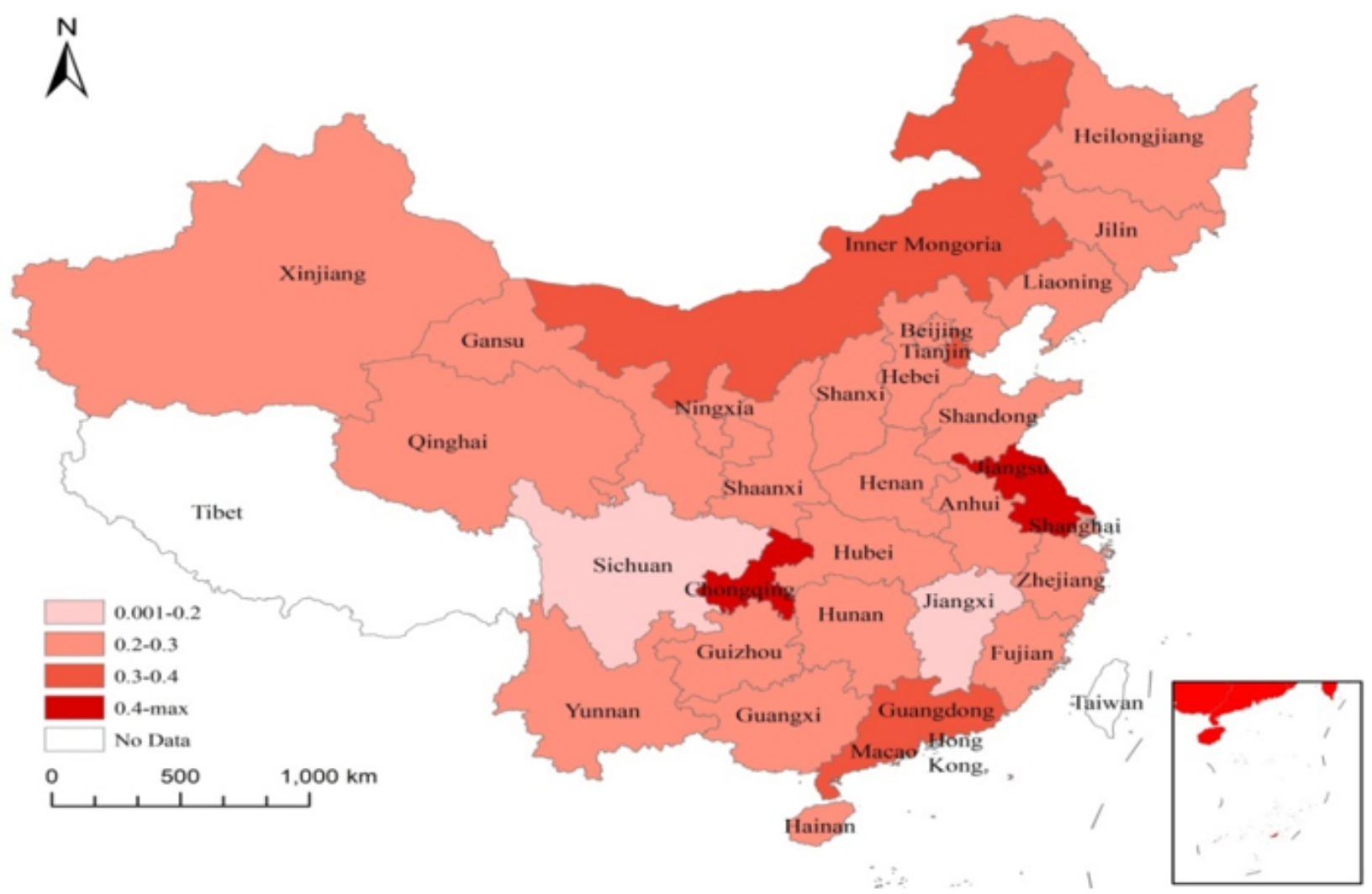

Figure 6 
Spatial distribution of dental fluorosis in mainland China during 1995-2020(created by ArcGIS 10.2 software). Note: The designations employed and the presentation of the material on this map do not imply the expression of any opinion whatsoever on the part of Research Square concerning the legal status of any country, territory, city or area or of its authorities, or concerning the delimitation of its frontiers or boundaries. This map has been provided by the authors. 